\title{
A CHARACTERIZATION OF PARETO SURFACES
}

\author{
LOUIS J. BILLERA ${ }^{1}$ AND ROBERT E. BIXBY ${ }^{2}$
}

\begin{abstract}
Given $n$ concave continuous functions $u_{i}$ defined over the unit $m$-cube $I^{m}$, the corresponding attainable set $V$ and Pareto surface $P$ are defined. In the economic interpretation, $V$ corresponds to the set of attainable utility outcomes realized through trading, and $P$ the set of such outcomes for which no trader can attain more without another getting less. Sets of the form of $V$ and $P$ are characterized among all subsets of $R^{n}$. The notion of complexity (the smallest $m$ for which a given $V$ can be realized) is briefly discussed, as is the idea of a "market game".
\end{abstract}

1. Introduction. The notion of an $n$-trader $m$-commodity pure trading economy is discussed by Shapley and Shubik in [6], where they characterize the class of "transferable utility market games" among all such games. It is our purpose to study in detail the class of market games without transferable utility (see, e.g., Scarf [5]). We restrict considerations to the class of markets discussed in [6], namely those having finitely many commodities and traders ( $m$ and $n$ respectively), and having the preferences of each trader given by a concave continuous utility function $u_{i}$.

In $\S 2$, we define the attainable set of utilities for a market, and the associated Pareto surface. The main result (Theorem 2.3) characterizes attainable sets as those sets of the form $C-R_{+}^{n}$ where $C$ is compact and convex. In fact, any such set can be realized by a market with $m=n(n-1)$ commodities and utility functions that are nondecreasing (in each variable).

In $\S 3$, we define the complexity of an attainable set $V$ to be the least number of commodities needed in a market realization of $V$. An example is given of a set having complexity equal to two. In $\S 4$, market games are

Presented to the Society, August 30, 1972; received by the editors January 11, 1973 and, in revised form, Fébruary 16, 1973.

AMS (MOS) subject classifications (1970). Primary 52A20; Secondary 90A15, 90D12.

Key words and phraises. Pareto surface, concave function, utility function, economic market, trading economy, game without side-payments, convex set.

1 Partially supported by the National Science Foundation under grants GK 29838 and GP 32314X.

${ }^{2}$ Partially supported by a National Science Foundation Graduate Fellowship and by National Science Foundation grant GK 29838 at Cornell University.

(c) American Mathematical Society 1973 
defined, and we show that every pure bargaining game is a market game needing at most $n^{2}$ commodities for a realization.

2. Attainable sets and Pareto surfaces. Let $I^{m}=[0,1]^{m}$ be the unit $m$-cube where $m$ is a positive integer. Suppose $u_{i}: I^{m} \rightarrow R$ are concave, continuous functions for $i=1, \cdots, n$.

Definition 2.1 The attainable set for $u_{1}, \cdots, u_{n}$ is the set

$$
V=\left\{x \in R^{n} \mid x_{i} \leqq u_{i}\left(y^{i}\right) ; y^{i} \in I^{m}, \sum_{i=1}^{n} y^{i}=e^{m}\right\}
$$

where $e^{m}=(1, \cdots, 1) \in I^{m}$. The Pareto surface for $u_{1}, \cdots, u_{n}$ is the set

$$
P=\{x \in V \mid y \in V, y \geqq x \Rightarrow y=x\} .
$$

It is easy to see that $V$ must be a closed convex set, and $P$ is included in the boundary of $V$. However, $P$ need not be closed. This will follow from our characterization of $V$ and from the example of [1, p. 89].

In the following, we will investigate some operations under which the class of attainable sets is invariant. If $f$ and $g$ are real valued functions on $I^{m}$ and $I^{l}$ respectively, then we define $f \oplus g$ and $f \wedge g$ from $I^{m+l}$ to $R$ as follows: if $(x, y) \in I^{m} \times I^{l}=I^{m+l}$, then $(f \oplus g)(x, y)=f(x)+g(y)$ and $(f \wedge g)(x, y)=f(x) \wedge g(y)=\min (f(x), g(y))$. Note that if $f$ and $g$ are continuous, concave and nondecreasing, then so are $f \oplus g$ and $f \wedge g$.

Lemma 2.2. Suppose $V_{1}$ and $V_{2}$ are attainable sets for $u_{1}, \cdots, u_{n}$ on $I^{m}$ and $w_{1}, \cdots, w_{n}$ on $I^{l}$ respectively. Let $b \in R^{n}, \alpha \in R$ and $\alpha>0$. Then

(2.2.1) $\alpha V_{1}+b$ is the attainable set for $\alpha u_{1}+b_{1}, \cdots, \alpha u_{n}+b_{n}$ on $I^{m}$,

(2.2.2) $\quad V_{1}+V_{2}=\left\{v_{1}+v_{2} \mid v_{1} \in V_{1}, \quad v_{2} \in V_{2}\right\}$ is the attainable set for $u_{1} \oplus w_{1}, \cdots, u_{n} \oplus w_{n}$ on $I^{m+l}$, and

(2.2.3) $\quad V_{1} \cap V_{2}$ is the attainable set for $u_{1} \wedge w_{1}, \cdots, u_{n} \wedge w_{n}$ on $I^{m+l}$.

Proof. We will prove only (2.2.3). Let $V$ be the attainable set for $u_{i} \wedge w_{i}, i=1, \cdots, n$. We will show $V=V_{1} \cap V_{2}$. A point $x \in V$ if and only if there exist $\left(y^{i}, z^{i}\right) \in I^{m+l}$ such that $\sum_{i=1}^{n}\left(y^{i}, z^{i}\right)=\left(e^{m}, e^{l}\right)$ and, for each $i$, $x_{i} \leqq\left(u_{i} \wedge w_{i}\right)\left(y^{i}, z^{i}\right)=u_{i}\left(y^{i}\right) \wedge w_{i}\left(z^{i}\right)$. This holds if and only if there exist $y^{i} \in I^{m}$ such that $\sum_{i=1}^{n} y^{i}=e^{m}$ and $z^{i} \in I^{l}$ such that $\sum_{i=1}^{n} z^{i}=e^{l}$, and, for each $i, x_{i} \leqq u_{i}\left(y^{i}\right)$ and $x_{i} \leqq w_{i}\left(z^{i}\right)$. This last statement is equivalent to $x \in V_{1} \cap V_{2}$.

We are now able to give a characterization of the class of attainable sets. We denote $R_{+}^{n}=[0, \infty)^{n}$.

THEOREM 2.3. $V \subseteq R^{n}$ is the attainable set for some concave continuous $u_{1}, \cdots, u_{n}$ on the unit $m$-cube $I^{m}$ for some $m>0$ if and only if $V=C-R_{+}^{n}$ where $\varnothing \neq C \subseteq R^{n}$ is compact and convex. Further, a representation can be given with $m \leqq n(n-1)$ and the $u_{i}$ nondecreasing. 
Proof. Suppose $V$ is the attainable set for $u_{i}, i=1, \cdots, n$, on $I^{m}$. It is easy to verify that $V=C-R_{+}^{n}$ where $C$ is the convex hull of $A$, and

$$
A=\left\{\left(u_{1}\left(y^{1}\right), \ldots, u_{n}\left(y^{n}\right)\right) \mid y^{i} \in I^{m}, \sum_{i=1}^{n} y^{i}=e^{m}\right\} .
$$

Since the $u_{i}$ are continuous, $A$ is compact and hence $C$ is compact and convex.

Conversely, suppose $V=C-R_{+}^{n}$. By (2.2.1), we may assume without loss of generality that $C \subseteq I^{n}$. We will adopt the following notation: if $x \in R^{n}$, then $x^{(i)}=\left(x_{1}, \cdots, x_{i-1}, x_{i+1}, \cdots, x_{n}\right) \in R^{n-1}$.

For each $i=1, \cdots, n$ let $^{3}$

and

$$
S_{i}=\left\{(a, \beta) \in R^{n-1} \times R \mid\left\langle x^{(i)}, a\right\rangle+x_{i} \leqq \beta \text { for all } x \in V\right\},
$$

$$
S_{i}^{\prime}=\left\{(a, \beta) \in S_{i} \mid \beta-\sum_{j \neq i} a_{j} \geqq 1-n\right\} .
$$

We define $f_{i}: I^{n-1} \rightarrow R$ by $f_{i}(y)=\inf \left\{\beta-\langle y, a\rangle \mid(a, \beta) \in S_{i}^{\prime}\right\}$ for $y \in I^{n-1}$.

Since $V$ is unbounded in all nonpositive directions, it follows that $(a, \beta) \in S_{i}$ implies $a \geqq 0$. Thus $f_{i}(y) \geqq 1-n$ for $y \in I^{n-1}$, i.e., $f_{i}$ is a finite concave function over $I^{n-1}$. By [4, Theorem 10.2, p. 84], we have that $f_{i}$ is lower semicontinuous on $I^{n-1}$. Since

$$
\begin{aligned}
\left\{(y, \mu) \in I^{n-1} \times R \mid \mu\right. & \left.\leqq f_{i}(y)\right\} \\
& =\bigcap_{(a, \beta) \in S_{i^{\prime}}}\left\{(y, \mu) \in I^{n-1} \times R \mid \mu \leqq \beta-\langle y, a\rangle\right\}
\end{aligned}
$$

is closed, it follows from [4, Theorem 7.1, p. 51] that $f_{i}$ is also upper semicontinuous. Also $f_{i}$ is nonincreasing since it is the infimum of nonincreasing functions.

Let $V^{i}$ be the attainable set of $u_{1}^{i}, \cdots, u_{n}^{i}$ on $I^{n-1}$ where for $y \in I^{n-1}$, and $j=1, \cdots, n$,

$$
\begin{aligned}
u_{j}^{i}(y) & =y_{j} & & \text { if } j \neq i \\
& =f_{i}\left(e^{n-1}-y\right) & & \text { if } j=i .
\end{aligned}
$$

It remains for us to show that $V=\bigcap_{i=1}^{n} V^{i}$, for in this case $V$ will be representable as the attainable set for continuous concave nonincreasing functions over $I^{n(n-1)}$ by (2.2.3).

To show $V \subset \bigcap_{i=1}^{n} V^{i}$, it is enough to show $C \subset V^{i}$ for each $i$. Let $x \in C$ and $i \in\{1, \cdots, n\}$. For all $(a, \beta) \in S_{i}^{\prime}, x_{i} \leqq \beta-\left\langle x^{(i)}, a\right\rangle$, and hence

$$
x_{i} \leqq f_{i}\left(x^{(i)}\right)=u_{i}^{i}\left(e^{n-1}-x^{(i)}\right) .
$$

${ }^{3}$ We will use the convention that if we are considering $S_{i}, S_{i}^{\prime}, f_{i}$ or $V_{i}$ then points $p$ in $R^{n-1}$ and $I^{n-1}$ are indexed $\left(p_{1}, \cdots, p_{i-1}, p_{i ! 1}, \cdots, p_{n}\right)$. 
Also, for $j \neq i$,

and so $x \in V^{i}$.

$$
x_{j}=u_{j}^{i}\left(0, \cdots, 0, x_{j}, 0, \cdots, 0\right),
$$

Now suppose $x \in\left(\bigcap_{i=1}^{n} V^{i}\right) \backslash V$. Then there exists $b \in R^{n}, \beta \in R$ such that $b \neq 0,\langle b, x\rangle>\beta$ and $\langle b, y\rangle \leqq \beta$ for all $y \in V$. Again, we must have $b \geqq 0$. Since we have assumed $C \subseteq I^{n}$, we must also have $\beta \geqq 0$.

If $i$ is such that $b_{i}>0$, then $\left(b^{(i)} / b_{i}, \beta / b_{i}\right) \in S_{i}$. Since $x \in V^{i}$, there must be a $y \in V^{i}$ such that $y^{(i)} \in I^{n-1}, y_{i}=f_{i}\left(y^{(i)}\right), y \geqq x$, and hence $\langle b, y\rangle>\beta$. Then

and hence

$$
\left\langle b^{(i)}, y^{(i)}\right\rangle+b_{i} f_{i}\left(y^{(i)}\right)>\beta,
$$

$$
f_{i}\left(y^{(i)}\right)>\beta / b_{i}-\left\langle b^{(i)} / b_{i}, y^{(i)}\right\rangle .
$$

Therefore, $\left(b^{(i)} / b_{i}, \beta / b_{i}\right) \notin S_{i}^{\prime}$, which implies

$$
\beta / b_{i}-\left(\sum_{j \neq i} b_{j}\right) / b_{i}<1-n .
$$

Thus for each $i=1, \cdots, n$,

$$
-\sum_{j \neq i} b_{j}<b_{i}(1-n) .
$$

Summing (2.3.1) over $i$ yields

$$
(1-n) \sum_{i=1}^{n} b_{i}<(1-n) \sum_{i=1}^{n} b_{i},
$$

which proves $\bigcap_{i=1}^{n} V^{i} \subset V$, and completes the proof of the theorem.

Corollary 2.4. $P \subseteq R^{n}$ is the Pareto surface for some concave continuous $u_{1}, \cdots, u_{n}$ on $I^{m}$ for some $m>0$ if and only if

(2.4.1) $x, y \in P, x \leqq y \Rightarrow x=y$,

(2.4.2) $P$ is bounded, and

(2.4.3) $P-R_{+}^{n}$ is closed and convex.

Proof. If $P$ is a Pareto surface, then (2.4.1) follows from (2.1.2). If $V=C-R_{+}^{n}$ is the associated attainable set, where $C$ is compact and convex, then $P \subset C$ and hence (2.4.2). Since $V=P-R_{+}^{n}$, we have also (2.4.3).

Conversely, suppose $P$ satisfies (2.4.1)-(2.4.3). Let $C$ be the closure of the convex hull of $P$. By (2.4.2), $C$ is compact and convex. Let $V=C-R_{+}^{n}$. Clearly $V \supset P-R_{+}^{n}$, and since $P-R_{+}^{n} \supset C$ by (2.4.3), also $P-R_{+}^{n} \supset V$. By the theorem, $V$ is an attainable set, and since $V=P-R_{+}^{n}, P$ must be the associated Pareto surface by (2.4.1). (In fact, the theorem tells us that we can find a representation with $m \leqq n(n-1)$ and the $u_{i}$ nondecreasing.) 
We note here that if the definition of attainable set is altered to require that each $u_{i}$ be the restriction of a concave, continuous function $g_{i}$ defined (and finite) over all of $R_{+}^{m}$, then Theorem 2.3 is still true. In fact, $g_{i}$ can be produced that are defined (and finite) over all of $R^{m}$. One can show this by changing the proof of Theorem 2.3 as follows. We may assume first that $C$ is contained in $\left[0, \frac{1}{2}\right]^{n}$. Now define

$$
S_{i}^{\prime \prime}=\left\{(a, \beta) \in S_{i}^{\prime} \mid \text { for some } x \in C,\left\langle x^{(i)}, a\right\rangle+x_{i}=\beta\right\} .
$$

Defining $\bar{f}_{i}: R^{n-1} \rightarrow R$ as we defined $f_{i}$, but with $S_{i}^{\prime \prime}$ in place of $S_{i}^{\prime}$, the proof will follow as before, providing we can show $\bar{f}_{i}(y)$ is finite for all $y \in R^{n-1}$. Since $\bar{f}_{i}$ is nonincreasing, it is enough to show $\bar{f}_{i}(y)$ is finite for all $y \in R_{+}^{n-1}$.

As before, $\bar{f}_{i}(y)$ is finite for $y \in I^{n-1}$. Let $y \in R_{+}^{n-1} \mid I^{n-1}$. Let $\langle a, \beta\rangle \in S_{i}^{\prime \prime}$, and suppose $x \in C$ is such that $\left\langle x^{(i)}, a\right\rangle+x_{i}=\beta$. Then $x^{(i)} \in\left[0, \frac{1}{2}\right]^{n-1}$ and $\beta-\left\langle x^{(i)}, a\right\rangle=x_{i} \leqq 1$. Let $\|p\|=\max _{i}\left|p_{i}\right|$ for $p \in R^{n-1}$. We have $\left\|x^{(i)}\right\| \leqq \frac{1}{2}$, and so $\left\|x^{(i)}+y / 2\right\| y\|\| \leqq 1$. Thus $x^{(i)}+y / 2\|y\| \in I^{n-1}$, and

$$
\beta-\left\langle x^{(i)}+y / 2\|y\|, a\right\rangle \geqq 1-n .
$$

Using this along with the fact that $\beta-\left\langle x^{(i)}, a\right\rangle \leqq 1$, we get $-\langle y, a\rangle \geqq-2\|y\| n$, and hence

$$
\beta-\langle y, a\rangle \geqq \beta-2\|y\| n \geqq-2\|y\| n .
$$

It then follows that $\bar{f}_{i}(y) \geqq-2\|y\| n$.

Complexity. Let $\mathscr{A}^{n}$ be the collection of all attainable sets in $R^{n}$. For $V \in \mathscr{A}^{n}$, we define the complexity of $V$ (com $V$ ) to be the least integer $m \geqq 0$ such that $V$ can be represented by $u_{i}$ 's over $I^{m}$ as in (2.1.1). Here we take $I^{0}=\{0\}$, and hence com $V=0$ if and only if $V$ can be represented with constant $u_{i}$ 's. It is easily seen that com $V=0$ if and only if $V=\{x\}-R_{+}^{n}$ for some $x \in R^{n}$.

If we define com $\mathscr{A}^{n}=\sup _{V \in \mathscr{S}^{n}} \operatorname{com} V$, we see from Theorem 2.3 that com $\mathscr{A}^{n} \leqq n(n-1)$. Clearly com $\mathscr{A}^{1}=0$ and it is easy to check that com $\mathscr{A}^{2}=1$.

In [3] the authors study, in particular, those $V \in \mathscr{A}^{n}$ with complexity equal to one. It is shown that com $V \leqq 1$ if and only if

$$
V=\left\{x \in R^{n} \mid h_{1}\left(x_{1}\right)+\cdots+h_{n}\left(x_{n}\right) \leqq a\right\}
$$

for $a \in R$ and $h_{i}: R \rightarrow R \cup\{\infty\}$ satisfying $\left\{(x, y) \in R^{2} \mid y \leqq-h_{i}(x)\right\}$ is an attainable set in $R^{2}$ for each $i$. In particular, each $h_{i}$ must be convex and nondecreasing. Using this characterization, geometrical necessary conditions for a set to have complexity one are derived.

As a result of the above discussion, one can see that $2 \leqq$ com $\mathscr{A}^{3} \leqq 6$. To show the lower bound we must exhibit a $V \in \mathscr{A}^{3}$ such that com $V>1$. 
Let $V=C-R_{\mathrm{i}}^{3}$ where $C$ is the convex hull of the set $\{(1,0,0),(0,1,0)$, $(0,0,1),(0,1,1)\}$.

A representation for $V$ over $I^{2}$ is given by $u_{1}(x, y)=x \wedge y, u_{2}(x, y)=y$ and $u_{3}(x, y)=x$. If $V$ had complexity equal to one, then there would exist nondecreasing, convex functions of one variable, $h_{1}, h_{2}, h_{3}$, and $a \in R$ such that

$$
V=\left\{\left(x_{1}, x_{2}, x_{3}\right) \mid h_{1}\left(x_{1}\right)+h_{2}\left(x_{2}\right)+h_{3}\left(x_{3}\right) \leqq a\right\} .
$$

One can assume that, for points on the Pareto surface of $V$, equality holds in the above expression. Thus $h_{1}(0)+h_{2}(1)+h_{3}(0)=a$ and $h_{1}(0)+h_{2}(1)+$ $h_{3}(1)=a$ which implies $h_{3}(0)=h_{3}(1)$. Now since $(1,0,0) \in V$ we must have $(1,0,1) \in V$, which is not the case. Thus the complexity of $V$ is two.

One of the authors is willing to conjecture that $\operatorname{com} \mathscr{A}^{n}=n-1$ for every $n$.

4. Market games. Suppose, in addition to the concave continuous function $u_{i}$ on $I^{m}$, we are given $\omega^{i} \in I^{m}$ for $i=1, \cdots, n$, where $\sum_{i=1}^{n} \omega^{i}=e^{m}$. Then if $\varnothing \neq S \subseteq N=\{1,2, \cdots, n\}$, we may define the attainable set for $S$ to be

$$
V(S)=\left\{x \in R^{S} \mid x_{i} \leqq u_{i}\left(y^{i}\right), i \in S ; y^{i} \in I^{m}, \sum_{i \in S} y^{i}=\sum_{i \in S} \omega^{i}\right\},
$$

where $R^{S}=\left\{x \in R^{n} \mid x_{i}=0\right.$ for $\left.i \notin S\right\}$. The function $V$ which associates with each $S$ the set $V(S)$ is called a market game (see [5] and [6]). From Theorem 2.3 it follows that each $V(S)$ is of the form $C_{S}-R_{+}^{S}$ where $C_{S} \subset R^{S}$ is compact and convex, and $R_{+}^{S}=R^{S} \cap R_{+}^{n}$.

In general, one can define a game to be any function $V$ on $2^{N}$ such that each $V(S)=C_{S}-R_{+}^{S}$, where $C_{S} \subseteq R^{S}$ is compact and convex. An interesting problem is to characterize the market games as a subset of all games.

In [6], Shapley and Shubik posed this problem while solving a related one. Given a market game $V$, they define a set function $v: 2^{N} \rightarrow R$ by

$$
v(S)=\max _{x \in V(S)} \sum_{i \in S} x_{i},
$$

and characterize these among all set functions on $2^{N}$.

In [2] the authors obtain a characterization of market games among the polyhedral games, i.e., games for which each $C_{S}$ is a polyhedron. The methods used there are special to polyhedra and do not seem to generalize. Unfortunately, the methods of this paper do not lend themselves to solving the problem for nonpolyhedral $V$. However, we can prove that a special class of games are market games using Theorem 2.3. We say a game $V$ is a pure bargaining (or unanimity) game if $0 \in V(N)$ while for each $S \neq N$, $V(S)=\{0\}-R_{+}^{S}$. 
THEOREM 4.1. Every pure bargaining game is a market game. Further there is a representation with $m \leqq n^{2}$ and nondecreasing $u_{i}$.

Proof. By Theorem 2.3, $V(N)$ is the attainable set for some $u_{1}, \cdots, u_{n}$ on $I^{l}$ for some $l \leqq n(n-1)$. Let $\omega^{1}, \cdots, \omega^{n} \in I^{l}$ be any points such that $\sum_{i=1}^{n} \omega^{i}=e^{l}$. Let $m=l+n$, and let $\hat{u}_{i}: I^{m} \rightarrow R$ be defined by $\hat{u}_{i}(y, x)=u_{i}(y) \wedge$ $n m_{i}\left(x_{1} \wedge x_{2} \wedge \cdots \wedge x_{n}\right)$ where $(y, x) \in I^{l} \times I^{n}=I^{m}$, and $m_{i}=\max _{x \in V(N)} x_{i}$. Finally, let $\hat{\omega}^{i}=\left(\omega^{i}, a^{i}\right)$, where $a^{i}$ is the $i$ th unit vector in $I^{n}$. Clearly, $\sum_{i \in N} \hat{\omega}^{i}=\left(e^{l}, e^{n}\right)=e^{l+n}$ and $V$ is the associated market game. Also we have $m \leqq n^{2}$.

For more on market games, see also [7].

\section{REFERENCES}

1. K. J. Arrow, E. W. Barankin and D. Blackwell, Admissible points of convex sets, Contributions to the Theory of Games, vol. II, Ann. of Math. Studies, no. 28, Princeton Univ. Press, Princeton, N.J., 1953. pp. 87-91. MR 14, 998.

2. L. J. Billera and R. E. Bixby, A characterization of polyhedral market games, Technical Report No. 177, Dept. of O.R., Cornell University, Ithaca, N.Y., 1973; Internat. J. of Game Theory (to appear).

3. — , Pareto surfaces of complexity 1, Tech. Rep., Dept. of O.R., Cornell University, Ithaca, N.Y. (forthcoming).

4. R. T. Rockafellar, Convex analysis, Princeton Math. Series, no. 28, Princeton Univ. Press, Princeton, N.J., 1970. MR 43 \#445.

5. H. Scarf, The core of an N-person game, Econometrica 35 (1967), 50-69.

6. L. S. Shapley and M. Shubik, On market games, J. Economic Theory 1 (1969), 9-25.

7. L. J. Billera, On games without side payments arising from a general class of markets, Tech. Rep. No. 184, Dept. of O.R., Cornell University, Ithaca, N.Y., 1973.

Department of Operations Research, Cornell University, Ithaca, New York 14850

Department of Mathematics, University of Kentucky, Lexington, Kentucky 40506 